\title{
Pilot study about the micro hydropower generation by use of flow induced vibration phenomenon
}

\author{
Yoshifumi Yokoi, ${ }^{1, *}$ \\ ${ }^{1}$ National Defence Academy of Japan, Department of Mechanical Engineering, 1-10-20 Hashirimizu, Yokosuka 239-8686, Japan
}

\begin{abstract}
In order to construct a micro power generation system using a piezo-electric element, power generation was tried using excitation oscillation of the bluff cylinder by the vortex shedding from the bluff cylinder. The bluff cylinder consists of a board spring section in which the piezo-electric element was attached, and a body section. The bluff cylinder was inserted into the water flow, the shape and the submersion depth of the bluff cylinder, and the flow velocity were varied, and the power generation characteristic was investigated. As a result, it was found that it can generate electricity by vortex excitation. It was found that the length and the submersion depth of the body section influence power generation. It was shown that the power generation characteristic changes with cross-sectional shape of the bluff cylinder. The most suitable state was the case where the submersion depth was set to $140 \mathrm{~mm}$ with a circular cylinder with a span length of $250 \mathrm{~mm}$. It is important to choose the power generation object which suited the use purpose.
\end{abstract}

\section{Introduction}

Considering an energy problem in recent years, it is interesting to exploit effectively the hydraulic power energy of the small scale and medium scale which is not yet used. As the example, the development and utilization in a river, an irrigation canal, etc. of low drop micro hydropower generation are performed actively. Those trends were reported by Turbomachinery Society of Japan $[1,2]$. In realization of the plan, there are many matters which cannot be suitable by extension of the technique developed in the large-scale water-power generation of the concentration of all aspect of a society in one place. Therefore, development of new power generation technique or some method is needed. In response to such background, the power generation method using the self oscillation (vortex excitation) by the vortex shedding from the bluff cylinder placed into the flow is proposed by Bemitsas et al. [3], Uno \& Kawashima [4], Koide et al. [5] and Hiejima et al. [6]. In order not to have a rotation mechanism the merit of vortex excitation type power generation is a maintenance-free. And the assembly and installation are also easy. Furthermore, since there is no impeller, power generation is possible, without damaging the living thing which lives in water by rotation of the impeller.

In this study, the piezo-electric element was used for the power generation element, and power generation was tried using excitation oscillation of the bluff cylinder by the vortex shedding from the bluff cylinder. Design manufacture of the power generation bluff cylinder was performed. And some tests were performed by the power generation bluff cylinder which was inserted into the water flow. In such tests, the shape and the submersion depth of the submersion section, and the flow velocity were varied, and the power generation characteristic was investigated.

\section{Experimental apparatus and method}

\subsection{Outline of experimental apparatus}

Experimental apparatus consists of a closed circuit water channel, a test bluff cylinder-traverse apparatus, and an oscillating frequency measuring device. The closed circuit water channel is the apparatus (water capacity 4 $\mathrm{m}^{3}$ ) used by the previous report [7]. The sizes of the test section were $2 \mathrm{~m}$ in length, $800 \mathrm{~mm}$ in width, and 430 $\mathrm{mm}$ in depth, the flow velocity range was $0-1.2 \mathrm{~m} / \mathrm{s}$, and the turbulence to the average flow velocity was less than $\pm 1.5 \%$. The test bluff cylinder-traverse apparatus is 2 axis dial slight movement type, and those moving ranges are $72 \mathrm{~mm}$ and $64 \mathrm{~mm}$, respectively. Those directions of moving are the depth direction and the width direction. As for the oscillation frequency measuring device, the storage oscilloscope (KENWOOD, DCS-7020) was used.

\subsection{Test cylinders and generators}

The schematic drawing of the test bluff cylinder (oscillating pendulum) is shown in Fig.1. The test bluff cylinder consists of a spring section (upper part) and a body section (lower part). The spring is a board with a length of $70 \mathrm{~mm}$, a width of $16 \mathrm{~mm}$, and a thickness of 2

* Corresponding author: yokoi@nda.ac.jp 


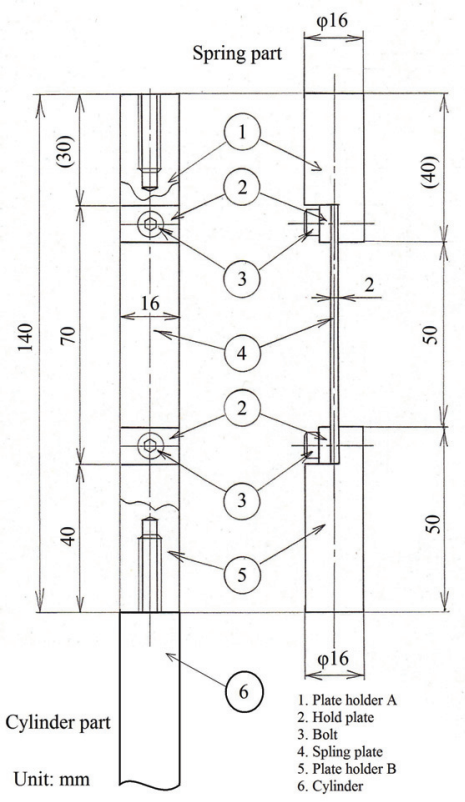

Fig. 1. The schematic drawing of a test cylinder spring section

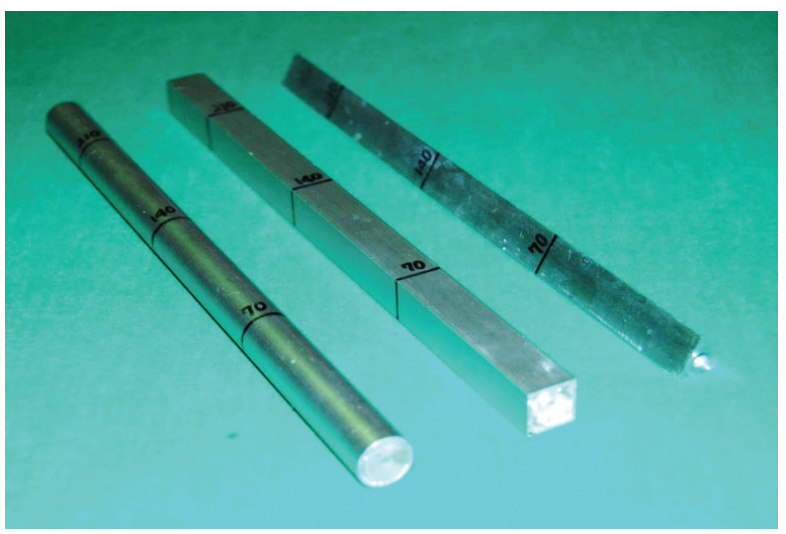

Fig. 2. Body section shape of the test cylinder (circular cylinder, square cylinder, and triangular cylinder)

$\mathrm{mm}$ made from vinyl chloride. As for the body, the circular cylinder, the triangular cylinder, and the square cylinder were prepared. Figure 2 shows the shape of those cylinders. The length of circular cylinder is two kinds, $250 \mathrm{~mm}$ and $500 \mathrm{~mm}$, and the diameter is $16 \mathrm{~mm}$. The length of a triangular cylinder and a square cylinder is $250 \mathrm{~mm}$, and those side lengths are $15 \mathrm{~mm}$.

The power generation object was set in the section of the board spring. The used power generation objects were shown in Fig. 3. One of which is a piezo-electric sensor (Tokyo sensor company, DT1-028 K/L) and the other is a piezo-electric buzzer (SPL company, PT08Z185). These two kinds of power generation objects are made by different material and the manufacture method.

\subsection{Experimental parameters}

Experiment parameters are the length of bluff cylinder, the submersion depth of the body (draft), its setting

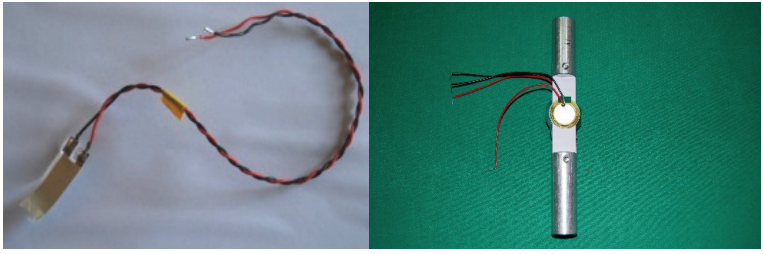

(a)

(b)

Fig. 3. The aspect of the power generation object, (a) piezo-electric sensor, (b) piezo-electric buzzer

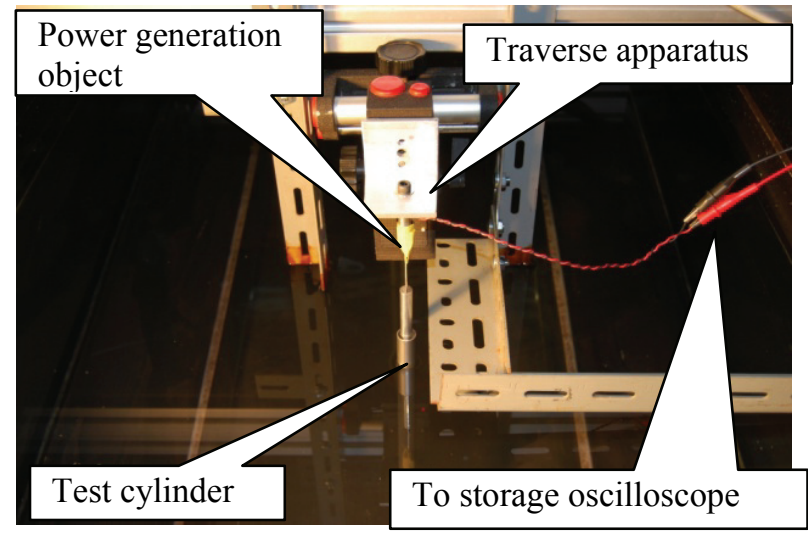

Fig. 4. The photograph of the situation where the test cylinder was set in the traverse apparatus

posture, and water flow velocity. The submersion depth of the body is three kinds $(70,140$ and $210 \mathrm{~mm})$. The posture of a triangular cylinder and a square cylinder is in the state where the angle was directed to the flow, and the state which directed the plane. The water flow velocity was varied to 44 steps in the range of $0-1.2 \mathrm{~m} / \mathrm{s}$.

\subsection{Experimental procedure}

The experiment procedure is the following. The power generation object is set in the board spring section of the test bluff cylinder, and the test bluff cylinder is attached in the traverse apparatus. The direction is decided to oscillate right-angled to the flow in the case of the attachment. The body is sunk in the target submersion depth. An aspect that it was set based on the procedure is shown in Fig. 4. The target flow velocity is caused by inverter operation of the pump of the closed circuit water channel. The voltage variation waveform from the power generation object sensor is observed with the storage oscilloscope, and output voltage and frequency are measured. Here, the frequency measured the signal of voltage waveform, observed the time jitter with the storage oscilloscope, and determined for fluctuation frequency. 
Tab. 1. Synthetic character frequency of the test cylinder

\begin{tabular}{|c|c|c|c|c|c|c|}
\hline \multicolumn{2}{|c|}{ Situation (mm) } & \multicolumn{5}{c}{ Cylinder shape (Hz) } \\
\hline span & draft & C.C. & S.S. & F.S. & S.T. & F.T. \\
\hline \multirow{2}{*}{250} & 70 & 2.5 & 2.0 & 1.9 & 2 & 2.2 \\
\hline & 140 & 2.2 & 1.7 & 1.7 & 1.9 & 2 \\
\hline \multirow{2}{*}{500} & 210 & 2.1 & 1.6 & 1.6 & 1.9 & \\
\hline & 205 & 0.9 & & & & \\
\hline
\end{tabular}

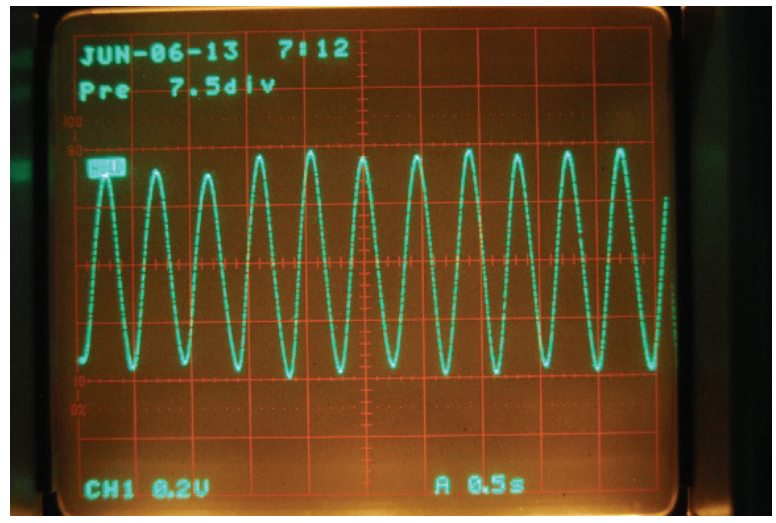

Fig. 5. An example of the output waveform from the power generation object

\section{Experimental result and discussion}

\subsection{Measurement of natural frequency}

The result of having measured the characteristic vibration of the overall system of the test bluff cylinder with a board spring is shown in Tab. 1. The meaning of the symbols used in the table are the following. The symbol "C.C." is a circular cylinder, the symbol "S.S." is the square cylinder which directed the angle to the flow, the symbol "F.S." is the square cylinder which directed the plane to the flow, the symbol "S.T." is the triangular cylinder which directed the angle to the flow, and the symbol "F.T." is the triangular cylinder which directed the plane to the flow. When the submersion depth of the body (draft) became shallow, it was confirmed that the character frequency as the overall system tends to become high.

\subsection{Power generation characteristic test}

Since the piezo-electric element reacts to unsteady stress, it can generate electricity by the lift alternately produced by the Karman vortex. An example of the power generation voltage waveform is shown in Fig. 5. In the beam which generally has a uniform section, if amplitude becomes large near critical oscillation

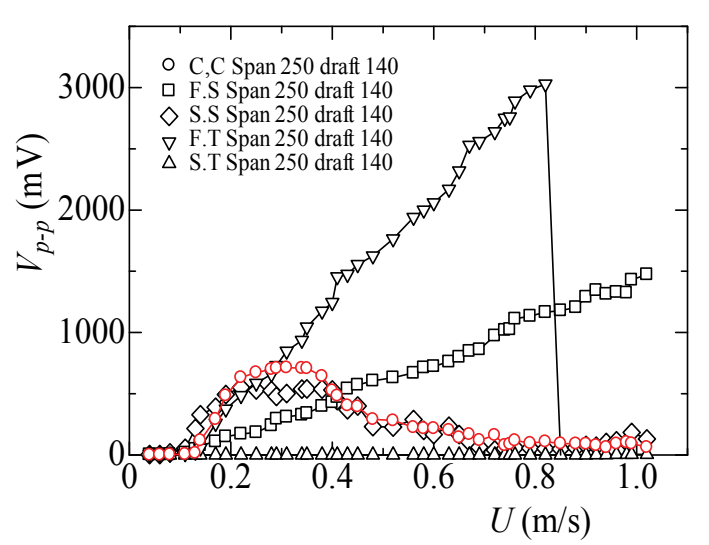

Fig. 6. Relationship between flow velocity and output voltage, in the case of circular cylinder

frequency and it passes over it, it is known that amplitude will become small. If the vortex excitation based on the Karman vortex shedding also makes the flow velocity increase from a halt condition, vortex shedding frequency also increases, and the bluff cylinder begins to oscillate based on critical oscillation frequency, and obtains a soon large amplitude point. Moreover, amplitude will become small if it passes over this point, and it is predicted that oscillation ceases. Figure 6 shows the relationship between the flow velocity and the produced voltage. An abscissa is the average flow velocity in the test section of the closed circuit water channel, and the ordinate is the average value of the peak-to-peak voltage observed by the oscilloscope waveform. In the case of the circular cylinder (C.C.) and the sharp square cylinder (S.S.), the number of peaks is one and after the peak with the increase in the flow velocity, the voltage shows a gently-sloping reduction. On the other hand, in the case of a flat triangular cylinder (F.T.) and a flat square cylinder (F.S.), there is no particular peak and the tendency which the voltage produced to the increase in the flow velocity is increasing is seen. In the case of a sharp triangular cylinder (S.T.), there was no response to the increase in the flow velocity. In the case of a circular cylinder and a sharp square cylinder, one peak with wide width can see. Since voltage is obtained even if the flow velocity changes, the peak with wide width is a thing desirable in use. Since the voltage of $500 \mathrm{mV}$ or more is obtained in 


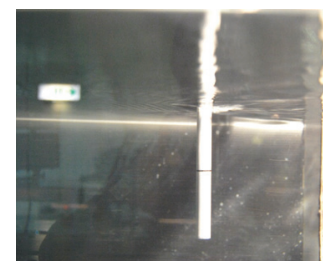

Side view

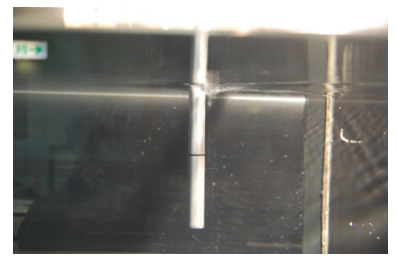

Side view

(b)
Side view

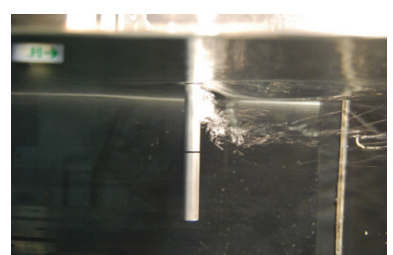

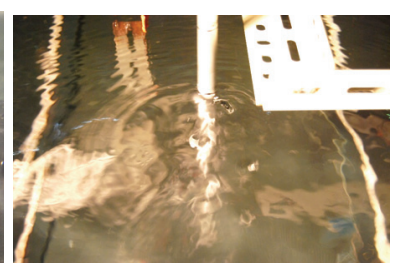

Bird 's-eye view

(a)

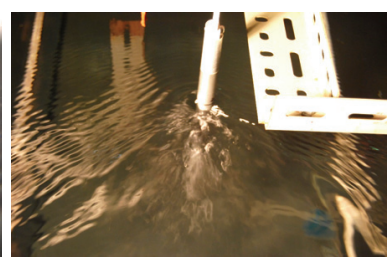

Bird 's-eye view

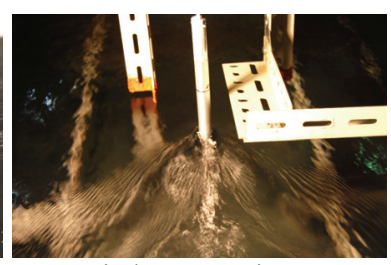

Bird 's-eye view (c)

Fig. 7. Flow feature around circular cylinder, (a) flow velocity; $0.13 \mathrm{~m} / \mathrm{s}$, (b) flow velocity; $0.38 \mathrm{~m} / \mathrm{s}$, (c) flow velocity; $0.79 \mathrm{~m} / \mathrm{s}$

the range of the flow velocity $0.22-0.38 \mathrm{~m} / \mathrm{s}$, considering this matter, as for the circular cylinder body and the sharp square body, it turns out that it is in the setting situation of having been most suitable for the use.

\subsection{The aspect of the flow around the bluff cylinder}

Figures 7 - 9 show the aspect of the flow of the circular cylinder, the square cylinder, and the triangular cylinder. The length of those bluff cylinders used here was $250 \mathrm{~mm}$. Figure 7 shows the aspect of the flow when sinking the circular cylinder $140 \mathrm{~mm}$ and varying the flow velocity. Where a right-hand side photograph is seen from the width side (underwater), a left-hand side photograph is in the state which looked at behind from slant. In Fig. 7(a), it is shown that the dimple of the water surface has produced alternately behind the circular cylinder. That shows that the Karman vortex is formed. The difference of a water level has not occurred between the upstream side of the circular cylinder, and the down stream side. In Fig. 7(b), the length which forms the dimple of the water surface of the circular cylinder behind becomes short, and an aspect that the wave is pulled from the circular cylinder can be seen. The difference of the water level can be seen have occurred between the upstream side of the circular cylinder, and the down stream side. As for this state, the value of the produced voltage becomes the maximum so

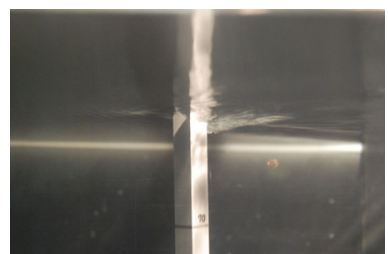

Side view

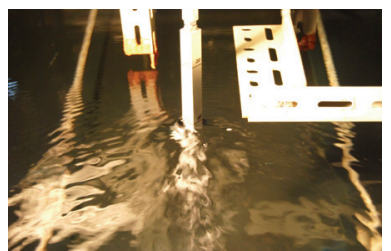

Bird 's-eye view

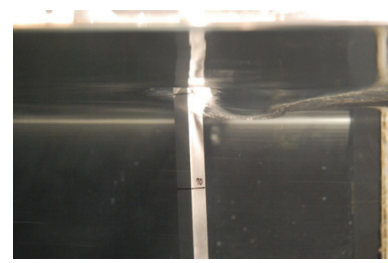

Side view

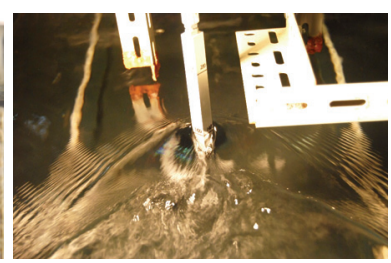

Bird 's-eye view (b)

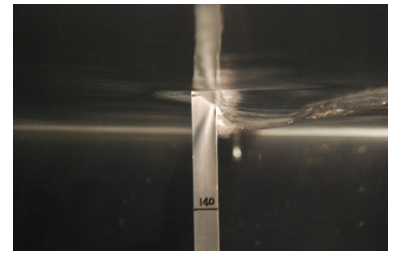

Side view

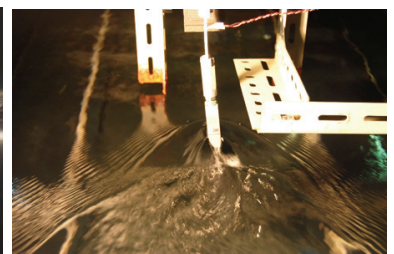

Bird 's-eye view

(c)

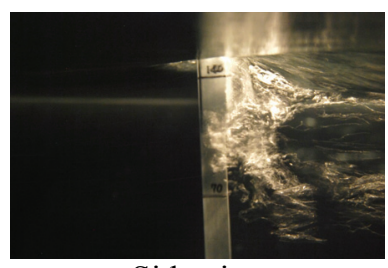

Side view

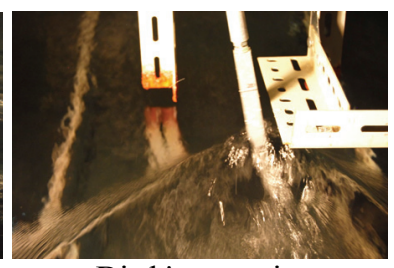

Bird 's-eye view (d)

Fig. 8. Flow feature around square cylinder, (a) sharp square cylinder (S. S.), flow velocity; $0.29 \mathrm{~m} / \mathrm{s}$, (b) sharp square cylinder (S. S.), flow velocity; $0.6 \mathrm{~m} / \mathrm{s}$, (c) Flat square cylinder (F. S.), flow velocity; $0.6 \mathrm{~m} / \mathrm{s}$, (d) Flat square cylinder (F. S.), flow velocity; $1.02 \mathrm{~m} / \mathrm{s}$

that may be seen in Fig. 5. In Fig. 7(c), the dimple of the water surface produced alternately is not seen, but the state where the towing wave has occurred from the circular cylinder is seen. The difference of the water level having occurred between the upstream side of the circular cylinder and the down stream side, and a cavity producing behind the circular cylinder, and involving in air bubbles is seen. This state is in the state in the middle of carrying out reduction with a gently-sloping value of the voltage produced in Fig. 5. Figure 8 shows the aspect of the flow when sinking the square cylinder $140 \mathrm{~mm}$ and varying the flow velocity. Figures 8(a) and 8(b) are in the state which directed the angle to the flow (S.S.), and those flow velocities are $0.29 \mathrm{~m} / \mathrm{s}$ and $0.6 \mathrm{~m} / \mathrm{s}$, respectively. In Fig. 8(a), generating of the Karman vortex is seen behind the square cylinder. And there are a few differences of the water level between the 


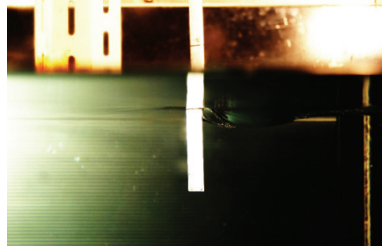

Side view

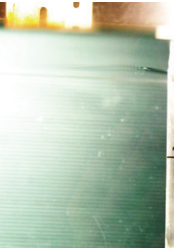

Side view

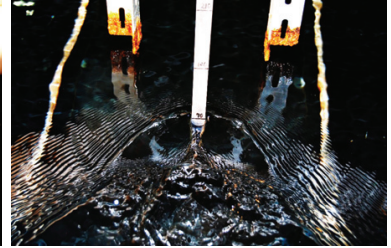

Bird 's-eye view

(a)

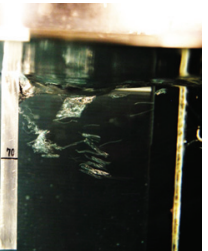

(b)

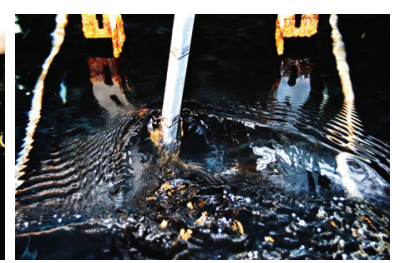

Bird 's-eye view
Fig. 9. Flow feature around triangular cylinder, (a) sharp triangular cylinder (S. T.), draft; $70 \mathrm{~mm}$, flow velocity; $0.6 \mathrm{~m} / \mathrm{s}$, (b) Flat triangular cylinder (F. T.), draft; $140 \mathrm{~mm}$, flow velocity; $0.6 \mathrm{~m} / \mathrm{s}$

upstream side of the square cylinder, and the down stream side. At this time, the value of the produced voltage was in the state which was a little less than the peak. On the other hand, generating of the Karman vortex is not seen by Fig. 8(b). An aspect that the towing wave has arisen from the square cylinder is shown. At this time, the difference of the water level occurs around the square cylinder, and the state where it became depressed from the water surface is remarkable. Figures $8(\mathrm{c})$ and $8(\mathrm{~d})$ are in the state which directed the face (side) to the flow (F.S.), and those flow velocities are 0.6 $\mathrm{m} / \mathrm{s}$ and $1.02 \mathrm{~m} / \mathrm{s}$, respectively. Formation of the Karman vortex is not seen behind of the square cylinder, but it is shown that the towing wave has arisen from the square cylinder. The difference of the water level between the upstream side of the square cylinder and the down stream side is also large, and involving in air bubbles is shown. Moreover, it is shown that the cellular involvement becomes intense with the increase in the flow velocity. This state does not exist at the self oscillation by the Karman vortex shedding, and the square cylinder is made for movement slowly carried out in a lift component direction to be carried out by the disproportionate flow which flows into the dimple produced behind square cylinder, and is considered that the voltage value produced with the increase in the flow velocity as shown in Fig. 6 increases in monotone. Figure 9 shows the aspect of the flow at the time of using the triangular cylinder. Figure 9(a) shows the state when making it sink $70 \mathrm{~mm}$, where the angle of the triangular cylinder is directed to the flow (S.T.), and setting the flow velocity as $0.6 \mathrm{~m} / \mathrm{s}$. The Karman vortex which should be formed behind the triangular cylinder is negated by interference of the towing wave, and is not seen. The difference of the water level exists between the upstream side of the triangular cylinder, and the down stream side. In the difference of this water level, it seems that it does not become what moves the triangular

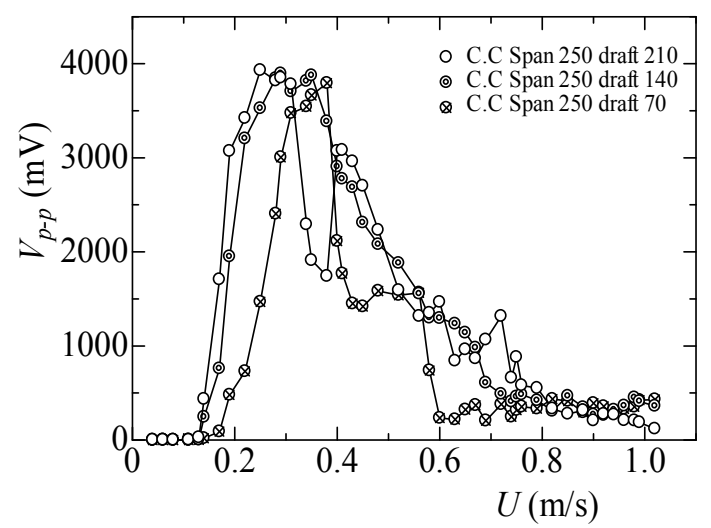

Fig. 10. Relationship between flow velocity and output voltage, in the case of circular cylinder, the piezo-electric buzzer was used

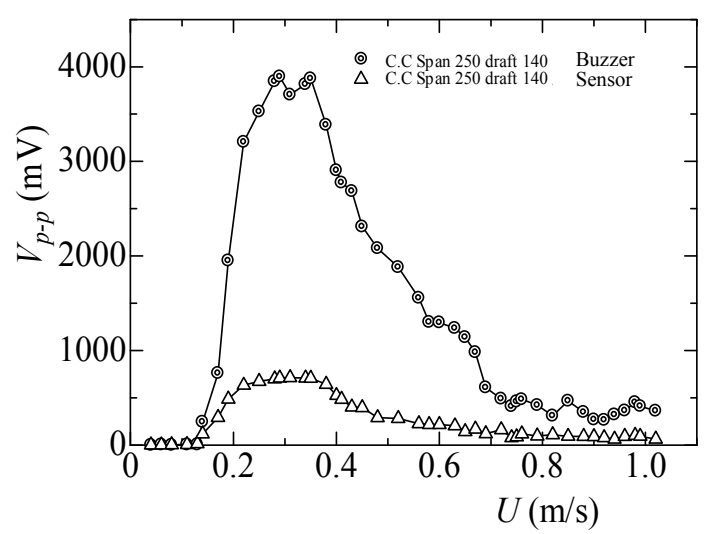

Fig. 11. Relationship between flow velocity and output voltage, in the case of circular cylinder, comparison of the piezo-electric sensor and the piezo-electric buzzer

cylinder since it has equal balance on both sides of the triangular cylinder in the case of the inflow to the down stream side. On the other hand, Fig. 9(b) shows the state when making it sink $70 \mathrm{~mm}$, where the side of the triangular cylinder is directed to the flow (S.T.), and setting the flow velocity as $0.6 \mathrm{~m} / \mathrm{s}$. Although formation of the Karman vortex is not seen behind the triangular cylinder, an aspect that the flat surface triangular cylinder is moving slowly greatly like the time of the flat surface square cylinder is seen. The difference of the water level in front and in rear of the triangular cylinder is also large, and an aspect that air bubbles are entangled in is shown. In the bluff cylinder which directed the "side" to the flow, although the Karman vortex was not produced behind the bluff cylinder, the bluff cylinder performed slowly large movement. The cause of that is because the flow which turns behind the bluff cylinder carries out unbalance on both sides. Therefore, since amplitude also becomes large by the increase in the flow velocity, the value of the voltage produced as shown in Fig. 6 also becomes large. 


\subsection{Comparison of power generation elements}

The experimental result when using a piezo-electric buzzer as the power generation object is shown in Fig. 10. In this experiment, using the circular cylinder with length of $250 \mathrm{~mm}$, the submersion depth of the body was set to $70,140,210 \mathrm{~mm}$, and water flow velocity was varied to 44 steps in the range of $0-1.2 \mathrm{~m} / \mathrm{s}$. Even when it changes into the piezo-electric buzzer, the power generation characteristic does not change, but since a case with a submersion depth of $140 \mathrm{~mm}$ of the body has wide peak width, it is thought that it is most suitable for the use. Figure 11 shows and compares the power generation voltage characteristic of two kinds of power generation objects (piezo-electric sensor and piezoelectric buzzer). Here, the circular cylinder of $250 \mathrm{~mm}$ length with submersion depth of $140 \mathrm{~mm}$ is used. In any case, the peak of power generation voltage is shown at the flow velocity range of $0.22-0.38 \mathrm{~m} / \mathrm{s}$. However, the clear difference is shown among both. It is shown that the voltage which a piezo-electric buzzer produces is about 4 times the voltage which a piezo-electric sensor produces. Since there is a difference on manufacture in the used product of two piezo-electric elements, it is guessed that such a result was obtained. It was found that it is important to choose the piezo-electric element corresponding to the purpose.

\section{Conclusions}

The following conclusions were obtained as a result of performing the characteristic test of the bluff cylinder pendulum aiming at generating electricity by vortex excitation.

(1) Generating of voltage was accepted by vortex excitation through the piezo-electric element.

(2) The length and the submersion depth of bluff cylinder influence the movement of the oscillating pendulum.

(3) The magnitude and its peak width of the voltage produced by vortex excitation corresponding to the flow velocity are changed by cross-sectional shape of the body section.

(4) The most suitable state was the case where the submersion depth was set to $140 \mathrm{~mm}$ with the circular cylinder with span length of $250 \mathrm{~mm}$.

(5) It is producing pendulum movement of the bluff cylinder, even when the balance of the inflow produced according to the difference of the water level collapses. In this case, the value of voltage continues increasing in monotone with the increase in the flow velocity.

(6) It is important to choose the power generation object which suited the use purpose.

Author is thankful to Mr. Jalernsk Kamon, Mr. Srisuntornsiri Rungsak and Mr. Kouichirou Ooue who helped this work and who are the student staff of my laboratory.

\section{References}

1. Turbomachinery Society of Japan ed., Turbomachinery, 30(3), 129 (2002)

2. Turbomachinery Society of Japan ed., Turbomachinery, 30(4), 193 (2002)

3. M. M. Bemitsas,K. Raghavan,Y. Ben-Simon,E. M. H. Garcia, Journal of Offshore Mechanics and Arctic Engineering, 130, 041101(2008)

4. M. Uno, R. Kawashima, Turbomachinery, 38(2), 121 (2010)

5. M. Koide, T. Sekizaki, S. Yamada, T. Takahashi, M. Shirakashi, Transactions of the Japan Society of Mechanical Engineers, 77(775), 702 (2011)

6. S. Hiejima, K. Oka, K. Hayashi, H. Inoue, Annual Journal of Civil Engineering in the Ocean, 69(1), 12 (2013)

7. Y. Yokoi, 57th International Conference of Machine Design Departments (ICMD2016), 175 (2016) 